\title{
Sarcoma de Ewing/Pnet primário pré-sacralk relato de caso
}

\section{Ewing Sarcoma/Pnet pre-sacral: case report}

Guilherme T. Batistão', Giovana F. M. Tiezzi' ${ }^{1}$, Ana Karina M. Salge², Débora T.R.S. Abate ${ }^{3}$, Janainna G. P. Olegário $^{4}$, Edgar L. da Silva Junior ${ }^{5}$, Antônio P. Pereira ${ }^{6}$, Susimary A. T.Padulla ${ }^{7}$, Renata C.Rossi e Silva ${ }^{8}$

\begin{abstract}
RESUMO
O sarcoma de Ewing é um tipo de tumor ósseo, de comportamento agressivo, que acomete principalmente indivíduos abaixo dos 30 anos e com predomínio no sexo masculino. A família de tumores de Ewing compreende um espectro de neoplasias de células neuroectodérmicas primitivas as quais são células embrionárias que migram da crista neural. Neste relato apresenta-se paciente do sexo masculino, 19 anos, admitido na emergência com quadro de lombalgia, fraqueza de membros inferiores, dificuldade de urinar e fratura de fêmur direito há um ano. Solicitada ressonância magnética da pelve e coluna lombosacra que evidenciaram lesão expansiva pré-sacral e lesões extramedulares e intradurais. Paciente submetido a exame anatomopatológico que evidenciou sarcoma de Ewing/Tumor Neuroectodérmico Primitivo. Realizado tratamento cirúrgico para descompressão medular e posterior radioterapia e quimioterapia.
\end{abstract}

Palavras chave: Sarcoma de Ewing. Diagnóstico por Imagem. Immunohistochemistry.

\begin{abstract}
Ewing's sarcoma is a type of bone tumor that has aggressive behavior, which mainly affects individuals below 30 years and predominantly in males. The family of tumors includes Ewing a spectrum of neuroectodermal of primitive cells tumors which are embryonic cells migrating from the neural crest. In this report we present a male patient, 19 years, admitted to the emergency room with back pain, weakness of the lower limbs, difficulty urinating and right femur fracture a year ago. Requested magnetic resonance imaging of the pelvis and lumbosacral spine showed a presacral mass lesion and extramedullary and intradural lesions. Patient has been subjected to the pathological examination that showed Ewing's sarcoma / primitive neuroectodermal tumor. Surgery for spinal decompression and subsequent radiotherapy and chemotherapy have been conducted.
\end{abstract}

Keywords: Sarcoma, Ewing. Diagnostic Imaging. Imuno-Histoquímica.

1. Graduando em Medicina; Universidade do Oeste Paulista (Unoeste), Presidente Prudente, SP

2. Enfermeira, Doutora em Patologia, Universidade Federal de Goiás, Goiânia, GO

3. Fisioterapeuta, Doutora em Patologia, Universidade Federal Fronteira do Sul, Chapecó, SC.

4. Biomédica, Doutora em Patologia, Faculdade Talentos Humanos, Uberaba, MG.

5. Médico, Especialista em diagnóstico por imagem - Unoeste

6. Médico, Especialista em Patologia, Hospital Regional de Presidente Prudente, Presidente Prudente, SP

7. Fisioterapeuta, Doutora em Ciências da Saúde, Faculdade de Ciências e tecnologia - UNESP, Presidente Prudente, SP.

8. Fisioterapeuta, Doutora em Patologia, - Unoeste.
Correspondencia

Dra. Renata Calciolari Rossi e Silva. Rua José Bongiovanni, 700 - Cidade Universitária CEP 19100-000: Presidente Prudente - SP.

Artigo recebido em 07/08/2014 Aprovado para publicação em 24/02/2015 


\section{Introdução}

O sarcoma de Ewing (SE) constitui um grupo de tumores de pequenas células redondas que se desenvolvem em tecidos moles e ossos, de comportamento agressivo, acometendo indivíduos abaixo dos 30 anos com predomínio no sexo masculino. ${ }^{1,2,3} \mathrm{~A}$ família de tumores de Ewing compreende um grupo de neoplasias de células neuroectodérmicas primitivas, cuja origem são as células embrionárias da crista neural. Dependendo do grau de diferenciação neural, podem ser denominadas por SE - quando indiferenciado, ou por Tumor neuroectodérmico primitivo periférico (PPNET - do inglês Peripheral Primitive Neuroectodermal Tumor), quando apresenta características de diferenciação neural. Esta família de neoplasias é causada por anormalidades cromossômicas $\mathrm{t}(11 ; 22)(\mathrm{q} 24 ; \mathrm{q} 12)$, que é encontrada em mais de $85 \%$ dos casos, onde estas translocações cromossomais podem resultar na criação de vários produtos de fusão de genes. ${ }^{1-4}$ Este tumor apresenta dificuldade diagnóstica, pois suas células neoplásicas se assemelham às encontradas no linfoma, rabdomiossarcoma, neuroblastoma e carcinoma avenocelular. ${ }^{5}$

O diagnóstico do SE depende, principalmente de imuno-histoquímica e testes moleculares/genéticos. O antígeno p30/32 MIC2, também referido CD99 é uma glicoproteína com alta expressão em células hematopoiéticas e também nas células dos tumores da família Ewing envolvido em processos de adesão celular. Esta glicoproteína pode ser identificada em quase todos os tumores desta família e é um marcador positivo que pode ser utilizado para o diagnóstico diferencial. A principal aplicação deste antígeno foi para o diagnóstico diferencial de tumores de pequenas células redondas na infância. O marcador é fortemente expressado no SE e no PNET. ${ }^{2,6}$

Sendo estes tumores altamente agressivos, o prognóstico do paciente é geralmente muito ruim e o tratamento adequado destes tumores malignos primários tem como objetivo a cura, em vez de tratamentos paliativos, sempre que possível, e podem incluir: cirurgia, radioterapia isolada ou em combinação com quimioterapia. ${ }^{7}$ Para um bom diagnóstico, o aspecto radiográfico do SE é bastante variável, com padrões associados ao tipo de osso envolvido. De modo geral, a tomografia computadorizada (TC) e a Ressonância magnética (RM) são úteis no estadiamento da lesão, na avaliação da presença de metástases e na monitoração da resposta à quimioterapia. ${ }^{1,8,9}$
O objetivo deste estudo é relatar o diagnóstico e o tratamento dos sarcomas, os quais devem ser feitos com eficiência e agilidade para que o prognóstico do paciente seja favorável à sua reabilitação.

\section{Relato}

Paciente do sexo masculino de 19 anos foi admitido no Hospital Regional (HR) de Presidente Prudente - SP dia 10/06/2010 com quadro de lombalgia, fraqueza de membros inferiores, dificuldade de urinar e fratura de fêmur direito há um ano. Foi submetido a exames de imagem que mostraram traumatismo raquimedular. Foi encaminhado ao tratamento cirúrgico, evoluindo com fraqueza nos membros inferiores. Retornou ao HR e ao exame físico apresentou-se em bom estado geral, sendo solicitados exames de imagem e anatomopatológico. Foi diagnosticado sarcoma de Ewing/PNET pré-sacral, encaminhado à cirurgia e ao tratamento complementar com quimioterapia e radioterapia.

Na ressonância magnética da pelve foi evidenciada grande lesão expansiva medindo aproximadamente, 9 x 5,3 cm, localizada anterior à coluna sacro coccígea apresentando componentes, predominantemente sólidos com sinal heterogêneo nas ponderações T1 e T2 sofrendo realce pelo meio de contraste paramagnético. Os contornos eram bem definidos em suas margens superior e inferior e anterior, não se infiltrando nos tecidos moles da região pélvica, apenas promovendo discreto deslocamento anterior das alças intestinais e da bexiga. (Figura 1A).

O exame ressonância magnética da coluna lombossacra evidenciou lesões expansivas extras medulares e intradurais, visualizadas nos níveis de L2 a S2, hipointensas na ponderação T1 e apresentando sinal intermediário na ponderação T2, sofrendo importante realce pelo meio de contraste paramagnético, determinando alargamento dos forames intervertebrais nos níveis citados, notadamente à esquerda nos níveis de L2-L3, L3-L4, L4-L5, e a direita no nível L5-S1. As lesões descritas determinaram discreta erosão óssea na região posterior dos corpos vertebrais de L5, S1 e S2 (Figura 1B).

No exame anatomopatológico, a macroscopia evidenciou vários fragmentos irregulares de tecidos, medindo em conjunto $3,0 \times 2,0 \mathrm{~cm}$, de coloração castanho-claro e consistência firme. Foi realizada a técnica de imuno-histoquímica utilizando-se a técnica da streptavidina-biotina e revelação com 3'3 
Diaminobenzidina (DAB). Os anticorpos utilizados foram CD99 (DAKO/ Clone 12E7), Ki-67 (Cell Marque/ Clone SP6), CD45 (DAKO/Clone PD26E-2B11), Sinaptofisina (Cell Marque) O resultado obtido foi: positividade para Ki-67 em 90\% das células (Figura
2A); células positivas pós imunomarcação com CD45 (Figura 2B); positividade para sinaptofisina (Figura 2C); células positivas para CD99 (Figura 2C). O perfil imuno-histoquímico integrado ao quadro histológico foi compatível com EWING/PNET.

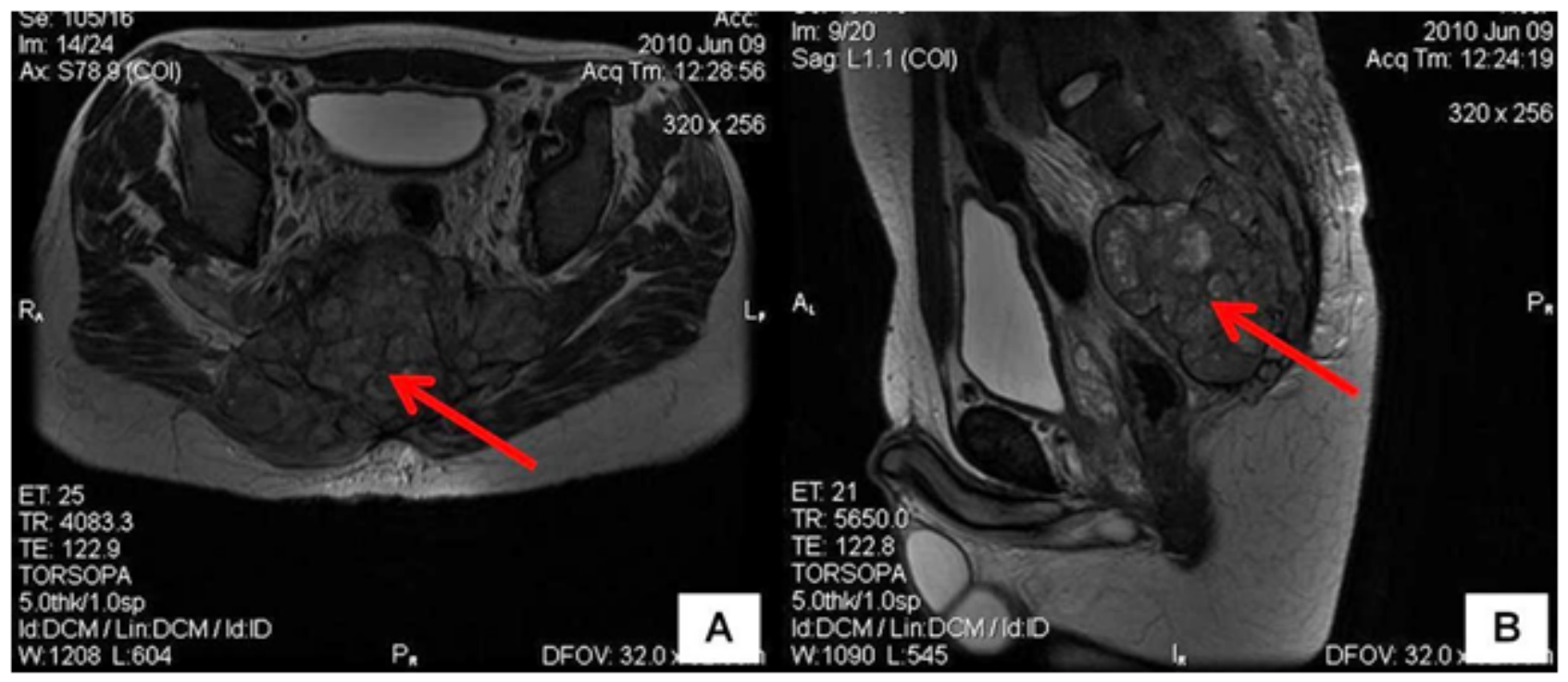

Figura 1: Em A: Ressonância magnética da pelve apresenta lesão expansiva com contornos lobulados acometendo a região sacrococcígea. Em B: Ressonância magnética da coluna lombo-sacra evidencia lesões expansivas extras medulares e intradurais, que determinam alargamento dos forames intervertebrais nos níveis de L2 e S2.

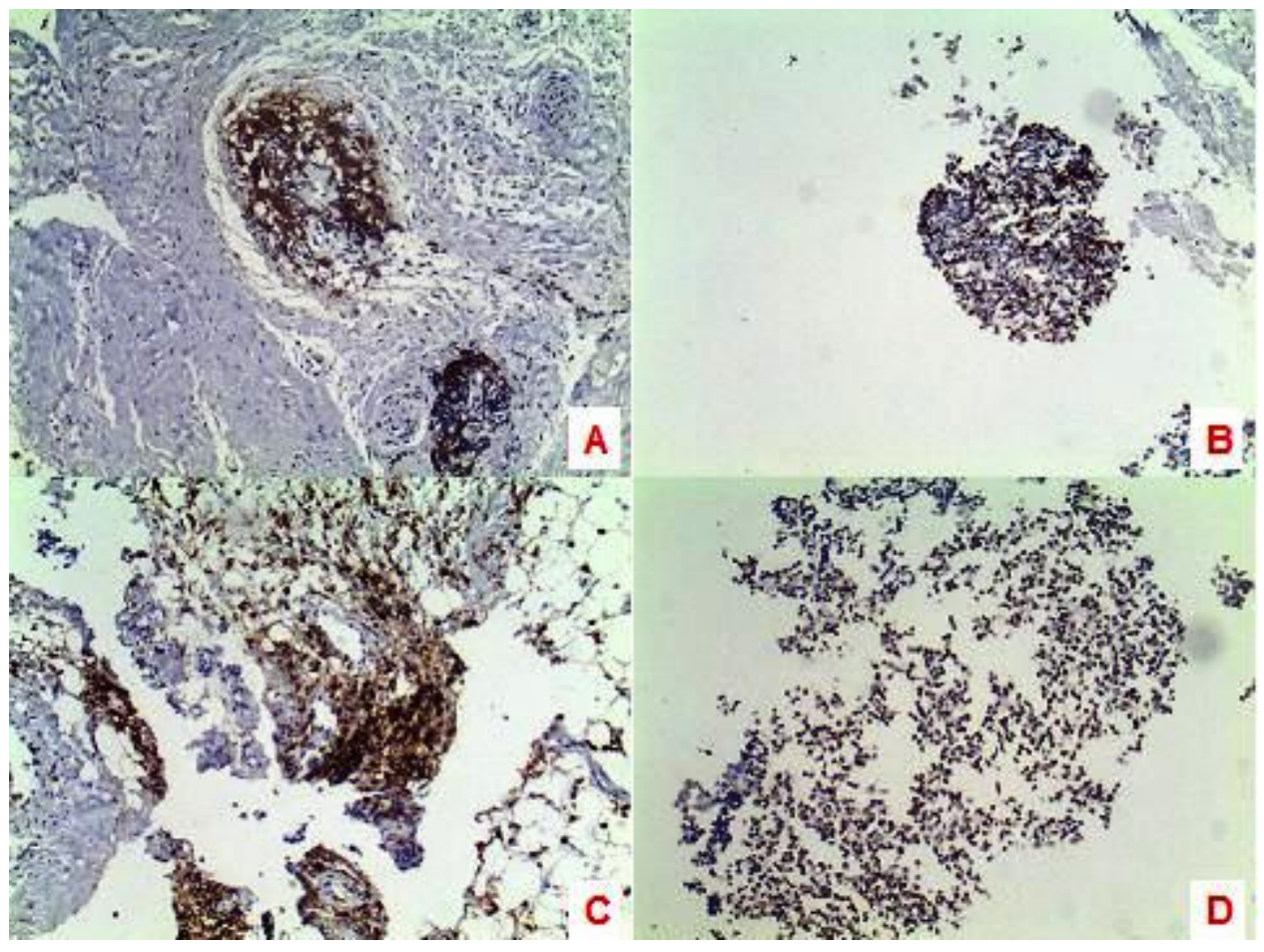

Figura 2: Imuno-histoquímica realizada para pesquisa de Sarcoma de Ewing. Em A positividade para Ki-67 em $90 \%$ das células; em B raras células positivas pós imunomarcação com CD45; em C positividade para sinaptofisina; em D raras células positivas para CD99. 


\section{Discussão}

O sarcoma de Ewing (SE) é um sarcoma de pequenas células redondas provenientes de tecidos moles, bem como do osso sendo um dos tumores malignos mais comuns em crianças e adultos jovens, podendo progredir rapidamente, apresentando um mal prognóstico. ${ }^{1,3}$ Nosso paciente tinha 19 anos, e era do sexo masculino condizendo com os estudos e os dados relatados na literatura. Relatamos as características morfológicas, resultados de exames de imagem e análise imuno-histoquimíca do tumor. Nossa análise histológica indicou que o paciente apresentava um tumor misto SE/PNET.

Ao exame por ressonância magnética $(\mathrm{RM})$ da coluna lombo-sacra, o paciente apresentou lesões expansivas extra-medulares e intradurais, que determinou alargamento dos forames intervertebrais nos níveis de L2 e S2. Este resultado, juntamente com a marcação positiva para o CD99, são úteis no diagnóstico diferencial ${ }^{1,2}$, e vão de encontro com os dados de outros autores, que relatam as principais lesões radiológicas e sua importância no diagnóstico precoce. ${ }^{1,9,12} \mathrm{O}$ comprometimento ilíaco ocorre em $18 \%$ dos tumores de Ewing, em 12\% dos osteossarcomas e $60 \%$ dos condrossarcomas mas são raros na infância e adolescência. ${ }^{13}$

O predomínio de esclerose ocorreu em todos os casos de osteossarcoma, também em metade dos casos de SE em um estudo desenvolvido em Vitória (ES). A presença de matriz osteóide é geralmente considerada indicativa de osteossarcoma. ${ }^{14}$

O linfoma apresenta uma sobreposição de características clínicas, radiológicas e até histológicas com o SE. Na maioria das vezes o linfoma linfoblástico é diferenciado pelo exame imunoistoquímico que se apresenta mais comumente positivo para CD45, e negativo para CD99 quando este não é possível ser usado na diferenciação pode-se utilizar a clinica ou diferenças citológicas. No caso apresentado o paciente apresenta um imuno-histoquimíco com padrão inverso ao mais comum do linfoma com CD99 positivo e CD45 raras células positivas. ${ }^{11}$

$\mathrm{Na}$ histologia, pode-se observar uma semelhança existente entre os diversos tumores de pequenas células redondas e para que haja um correto diagnóstico, a imunorreatividade para CD99 se faz necessária. Nosso paciente apresentou exame imunohistoquimíco positivo para CD99, este marcador pode ser identificado em, aproximadamente, todos os tumores da família dos $\mathrm{SE}^{10}$, constituindo um marcador positivo no estabelecimento de diagnóstico diferencial entre os tumores da família SE e outros tumores de pequenas células redondas. ${ }^{1,2}$ Foram utilizados também outros marcadores como Ki-67 que foi positivo em $90 \%$ das células, sinaptofisina positivo e CD45 com raras células positivas, importante para o diagnóstico diferencial com linfomas. ${ }^{11} \mathrm{~A}$ análise anatomopatológica da lesão extramedular, com perfil imuno-histoquimíco integrado ao quadro histológico é compatível com SE/PNET. O estudo imunohistoquimíco é imprescindível para que se determine a etiologia dos sarcomas da família Ewing e o seu diagnóstico.

O tratamento apropriado para SE tem sido a excisão cirúrgica do tumor associado à radioterapia $\mathrm{e}$ à quimioterapia. Tumores compreendidos entre 8-10 $\mathrm{cm}$ em diâmetro e tumores de eixo central (ex. pelve) exibem uma taxa alta de falha ao tratamento. ${ }^{15,16} \mathrm{Com}$ relação ao PNET não há consenso na literatura quanto ao tratamento. No nosso caso, como o tumor era misto, ou seja, um tumor raro e de difícil tratamento, após analisar as possibilidades de tratamento, optouse pela excisão cirúrgica. O tratamento cirúrgico é útil para descompressão medular e para o diagnóstico histopatológico. ${ }^{12}$

\section{Conclusão}

Para o diagnóstico precoce e correto, excluindo a variedade de diagnósticos diferenciais, os exames de imagem e histopatológicos, principalmente os marcadores imuno-histoquimicos como o CD99, são imprescindíveis, pois além da utilidade para visualização e classificação do tamanho das áreas de lesão, permitem uma avaliação e intervenção cirúrgica realizada de maneira mais efetiva ao paciente.

\section{Referências}

1. Catalan J, Fonte AC, Lusa JRB, Oliveira AD, Melo ES, Gonçalves CM. Sarcoma de Ewing: aspectos clínicos e radiográficos de 226 casos. Radiol Bras. 2005;38:333-6.

2. Pereira RJS. Atlas de Ortopedia e Traumatologia Clínica. São Paulo: látria; 2006.

3. Romero R, Abelairas J, Sanz J, Ruiz MM, Sendagorta E. [Recurrence ofperipheral primitive neuroectodermal tumor of the orbit with systemic metastases]. Arch Soc Esp Oftalmol. 2006;81:599-602. Spanish.

4. Pardini Jr A, Souza JMG. Clínica Ortopédica - tumores do sistema músculoesquelético. Rio de Janeiro: Editora Medsi; 2002. 
5. Abul KA, Nelson F, Vinay K. Robbins \& Cotran. Patologia Bases Patológicas das Doenças. 8ª Ed, p. 1361; 2010.

6. Leong AS-Y, Cooper K, Leong FJW-M. Manual of Diagnostic Antibodies for Immunohistology. 1999, Greenwich Medical Media Ltd, London. P. 115-6.

7. Leandro I. Dini, Rodrigo Mendonça, Pasquale Gallo. Primary ewing's sarcoma of the spine, Arq Neuropsiquiatr. 2006;64(3A):654-9

8. JLKaspers GJ, Kamphorst W, Graaff M, H Alphen AM, Veerman AJP. Primary spinal epidural extraosseous Ewing's sarcoma. Cancer. 1991;68:648-54.

9. Mirra JM, Picci P. Ewing's sarcoma. In bone tumors: clinical,radiologic, and pathologic correlations. New York: Lea\&Febiger, 1989:1088-117.

10. Scotlandi K, Baldini N, Cerisano V, Manara MC, Benini S, Serra M et al. CD99 engagement: an effective therapeutic strategy for Ewing tumors. Cancer Res. 2000;60:5134-42
11. Costa V, Oliva T, Almeida M, Norton L. Sarcoma de Ewing ou Linfoma ósseo. Revista Acta Pediatrica Port, vol. 36, № 6 p, 304-7, 2005

12. Spaziante R, Divitiis E, Giamundo A, Gambardella A, Di Prisco B.Ewingís sarcoma arising primarily in the spinal epidural space: fifthcase report. Radiol Bras. . 1983;12:337-41.

13. Schajowicz F. Neoplasias ósseas e lesões pseudotumorais. $2^{\mathrm{a}}$ ed. Rio de Janeiro: Revinter, 2000.

14. Oliveira GA, Werlang H Z, Bergoli P M, Frechiani M, Oliveira F. Tomografia computadorizada na análise dos padrões de calcificações nos tumores ósseos da bacia em pediatria: nova abordagem. Radiol Bras. 2006; 39: 413-18.

15. Horowitz ME, Tsokos MG, DeLaney TF. Ewing's sarcoma. CA Cancer J Clin. 1992; 42:300-20.

16. Telles NC, Rabson AS, Pomeroy TC. Ewing's sarcoma: an autopsy study. Cancer. 1978;41:2321-9. 